\title{
A Data-Driven Method Towards Minimizing Collision Severity for Highly Automated Vehicles
}

\author{
Masoumeh Parseh, Fredrik Asplund, Lars Svensson, Wolfgang Sinz, Ernst Tomasch, and Martin Törngren
}

\begin{abstract}
The deployment of autonomous vehicles on public roads calls for the development of methods that are reliably able to mitigate injury severity in case of unavoidable collisions. This study proposes a data-driven motion planning method capable of minimizing injury severity for vehicle occupants in unavoidable collisions. The method is based on establishing a metric that models the relationship between impact location and injury severity using real accident data, and subsequently including it in the cost function of a motion planning framework. The vehicle dynamics and associated constraints are considered through a precomputed trajectory library, which is generated by solving an optimal control problem. This allows for efficient computation as well as an accurate representation of the vehicle. The proposed motion planning approach is evaluated by simulation, and it is shown that the trajectory associated with the minimum cost mitigates the collision severity for occupants of passenger vehicles involved in the collision.
\end{abstract}

Index Terms-Motion planning, collision severity, data-driven, impact location, injury severity, trajectory library, occupant safety, optimal control.

\section{INTRODUCTION}

$\mathbf{T}$ HE automotive industry is greatly investing in the deployment of autonomous vehicles on public roads [1]; however, a major challenge of this deployment is ensuring vehicle safety in critical traffic situations. In the literature, various motion planning techniques have been developed to improve the safety of autonomous vehicles by providing contingency maneuvers. These methods either bring the vehicle to a safe stop [2], [3], [4], [5] or provide one or several parallel trajectories dealing with multiple outcomes [6], [7]. However, the best practice techniques applied in their implementation cannot always ensure an avoidance maneuver, for instance, due to limitations in vehicle modeling or the perception systems [8]. It is thus not surprising that the testing of autonomous vehicles on public roads has to date involved several accidents [9]. In fact, even with the most advanced autonomous vehicles developed by leading automotive manufacturers, accidents still occur [4]. Therefore, unavoidable collision situations are likely to exist and require the attention of vehicle manufacturers in the foreseeable future.

Manuscript received June 04, 2020; revised November 06, 2020; accepted February 16, 2021. This work is supported by ECSEL PRYSTINE project with grant agreement 783190 . Lars Svensson is funded by AutoDrive project, H2020-ECSEL. (corresponding author: Masoumeh Parseh).

M. Parseh, F. Asplund, L. Svensson and M. Törngren are with the Mechatronics and Embedded Control Systems Division, Department of Machine Design, KTH Royal Institute of Technology, Stockholm, Sweden. Email: \{mparseh, fasplund, larsvens, martint\}@kth.se.

W. Sinz and E. Tomasch are with Vehicle Safety Institute, Graz University of Technology, Graz, Austria. Email: \{ernst.tomasch,wolfgang.sinz\}@tugraz.at.
Motion planning in scenarios where a collision cannot be avoided has gained attention only recently. The authors of [10] developed a model predictive control (MPC)-based motion planner that outputs a trajectory with the lowest crash severity if avoidance is not possible. The crash severity is part of the formulation of the MPC cost function and is based on relative speed, relative heading angle and mass ratio between the vehicles. In [11], a crash severity map was developed by finite elements (FEM) simulations that aimed at identifying a crash configuration minimizing the crash severity. The method searches for a maneuver that leads to this crash configuration, which is determined by lateral offset and the relative heading angle. The severity of a crash in [11] is based on the deformation of nodes in the passenger compartment.

The main contribution of this paper is a data-driven method that estimates and utilizes collision severity in scenarios where a collision cannot be avoided. This method can initially rely on data sets that disregard heterogeneity (variations in vehicle types and collision environment). However, the method can be extended and improved by refining these data sets over time without affecting the original proposal. We thus contribute a method with the aim of avoiding the simplifications and assumptions that might be required by deductive approaches, both during design and run-time. We show the applicability of the method using real accident data by coupling it to a trajectory library planning approach that enables an accurate representation of the vehicle dynamics in aggressive maneuvers as we proposed in [12]. Our method provides improved accuracy in terms of collision severity representation, which enables the planner to further minimize the severity of the accident.

The rest of the paper is organized as follows. Section II presents related work in two research fields, i.e., accident data analysis and motion planning in critical scenarios. Section III characterizes the problem to be solved in terms of a choice between several feasible trajectories. Sections IV, V and VI together present a novel solution to the problem statement, with each section describing one aspect. In Section IV, we analyze accident data and define a data-driven metric that maps impact location to accident severity. In Section V, we introduce the trajectory library motion planning framework, in which the severity metric from Section IV can be included as a cost term. The integrated method for severity minimization motion planning is described in Section VI. The proposed solution is verified through simulations in Section VII. The paper concludes with discussion, and conclusions and future work, in sections VIII and IX, respectively. 


\section{RELATED WORK}

Planning a trajectory for a vehicle in unavoidable collision scenarios involves identifying the factors that contribute to the injury severity and understanding the techniques and requirements of motion planning in such critical situations. Below, we discuss the relevant work related to these concerns.

\section{A. Accident Data and Analysis}

Data from road vehicle accidents has been gathered and analyzed for a long time and for many different purposes. For example, researchers have studied collisions in order to build algorithms for injury severity predictions since the 1990s. Malliaris [13] identified more than twenty crash predictors that can estimate the likelihood of collisions leading to fatalities or at least one injury severity of the Maximum Abbreviated Injury Scale (MAIS) 3+ [14], [15] and one injury severity of MAIS +2 in highway accidents. Using the same accident data sources, Kononen et al. developed a model to predict the probability that a vehicle involved in a collision contains occupants with serious injuries [16]. The inputs for that model were delta-v (total change in the vehicle's velocity), direction of impact (front, left, right and rear), vehicle type, single or multiple impacts, seat belt use, age and gender. The conclusions were that delta-v, seat belt use and direction of impact influence the injury severity the most; left impacts are associated with the highest risk, followed by right, front and rear impacts [16]. Studies based on other accident data sources have arrived at the same result [17], [18], [19]. As an example, Lubbe et al. [18] plotted the risk of severe injury against delta-v for different accident types. They identified that as delta-v increases, a belted, single near-side collision has higher severity in comparison to a belted, single front or rear collision.

Similarly, Nishimoto et al. [19] identified the most important risk factors as delta-v, vehicle impact location, seat belt use, single/multiple impact, vehicle size and the age and seating positions of occupants. Furthermore, the injury risk was highest for the near side, followed by the far side, front and rear [19]. Huang et al. [20] performed an analysis to investigate the relationship between the point of impact and injury severity, and the results showed that the front passenger side and driver side impose higher risk to the driver in comparison to the rear passenger sides and rear corners of the vehicle.

Nevertheless, it should be noted that many factors risk confounding accident analysis, meaning that less conservative conclusions are associated with large uncertainties. As an example, severity data is often based on subjective assessments from police reports [21], which can differ significantly from objective measures of severity [22]. Similarly, behavioral differences between drivers and the under-reporting of less severe injuries can substantially skew conclusions from studies of accidents [21]. The existence of unobserved heterogeneity (variations in the effect of variables across the sample population that are unknown to the analyst) is especially concerning [23]. Although automated vehicles might mitigate much of this problem in regard to driver behavior, it will continue to exist for the foreseeable future due to the large heterogeneity of environmental factors and vehicle designs [23]. In fact, the problem will most likely persist at least until significant improvements in the sensors carried by automated vehicles enable them to provide data far surpassing what is available today [21].

In other words, it appears that we are limited to conservative conclusions based partly on logical reasoning when differentiating between trajectories for a highly automated vehicle in an inevitable collision scenario. As an example, the design of road vehicles in regard to absorbing the energy from collisions means that front and rear collisions are likely to be less severe than side collisions [16], [17], [18], [19], [24].

\section{B. Motion Planning in Critical Situations}

Motion planning in critical scenarios requires the vehicle to operate close to its physical limitations under tight time constraints. The motion planning algorithm needs an accurate representation of the vehicle to enable full utilization of its motion capability while ensuring that the real vehicle is able to track the planned trajectory. In addition, the execution time must be sufficiently short so that the vehicle can react to a dynamically changing environment [25].

These combined requirements make motion planning in critical situations a challenging problem. However, there have been several research efforts presenting real-time motion planning algorithms capable of planning motions approaching the physical limits by utilizing methods from numerical optimization [26], [27], [28]. With this approach, the motion planning problem is formulated as an optimal control problem that is solved online, where the vehicle dynamics and desired behavior are encoded as costs and constraints [29]. The optimization-based approach is advantageous for planning aggressive motions, as it scales comparatively well with the model complexity and therefore allows an accurate representation of the vehicle. It also allows for explicit encoding of the dynamic constraints of the vehicle [25]. However, since the optimization problem is solved online, the problem to be solved at each iteration needs to be formulated in a way that allows efficient solving, e.g. as a quadratic program [10], [26], [27]. The cost function of a quadratic program must be a quadratic function of states and controls. This requirement places restrictions on what desired behaviors can be expressed.

In the context of severity minimization motion planning, optimization-based methods prohibit a detailed representation of how the impact location affects the severity of the crash. For example, in [10], the crash direction is included in the severity index, represented by the relative heading angle between the vehicles, but the distinction between different impact locations is not included.

Sampling-based motion planners are generally less restrictive in this regard. The trajectory roll-out family of algorithms build on the principle of rolling out a set of candidate trajectories starting at the current vehicle state and selecting the optimal candidate using a cost function that can be formulated with very few restrictions. The practicality of the method has been proven in several studies [30], [31]. The efficiency of the method hinges on a fast method to generate the candidate trajectories. Werling et al. [31] proposed using quintic and quartic polynomials to represent lateral and longitudinal motion, and showed that this 
constitutes minimum jerk trajectories for the vehicle. Such trajectories can be computed efficiently, but are not suitable for dynamic maneuvers [2]. Hence, for trajectory roll-out methods there is a trade-off between computational efficiency and vehicle model accuracy.

One way to circumvent this trade-off is to move computations offline. By precomputing a library of trajectories using an accurate model, the online computation is reduced to selecting the optimal trajectory in the library. This approach has been successfully employed in many robotic applications, for example, the Mars exploration rover [32], evasive maneuvers for autonomous helicopters [33] and automated vehicles [2].

\section{Problem Statement}

We tackle the problem of reducing post-impact injury severity for passenger car occupants by controlling the vehicle maneuvers prior to an unavoidable collision. Such a severity minimization motion planning problem can be expressed as the following optimization problem:

\section{minimize accident severity \\ subject to vehicle dynamic constraints, initial condition.}

The optimal trajectory is the sequence of states, which minimizes the severity of the resulting accident while adhering to the dynamic limitations of the vehicle. As stated in Section II.A, the impact location is strongly correlated to the severity of an accident and should therefore be included in the cost function.

Due to the limitations of optimization-based methods in terms of cost function expressivity, as described in Section II.B, using such a method requires severe simplifications that may in turn lead to a loss of optimality with respect to the original problem. Therefore, we apply a trajectory library approach [2] to tackle the severity minimization problem.

The question to be answered is how the ego vehicle should select and execute a trajectory from the library to achieve minimum collision severity. To answer this question, we elaborate on how to establish the relationship between the impact location and collision severity in Section IV, and formulate an optimal control problem to generate the trajectory library in Section V. Finally, we construct a method for selecting a trajectory that minimizes the collision severity in Section VI.

\section{Collision Severity Analysis}

We analyzed real accident data to understand the relationship between the impact location and collision severity. In the following subsections, we introduce the accident data source, the associated filter criteria, and vehicle impact location classification we have used. Consequently, we estimate the risk associated with collisions with different vehicle locations.

\section{A. Data Source and Filter Criteria}

Studies show that collisions at intersections constitute more than $50 \%$ of all accidents, and their number has increased faster than other types of accidents over the years [35]. Furthermore,

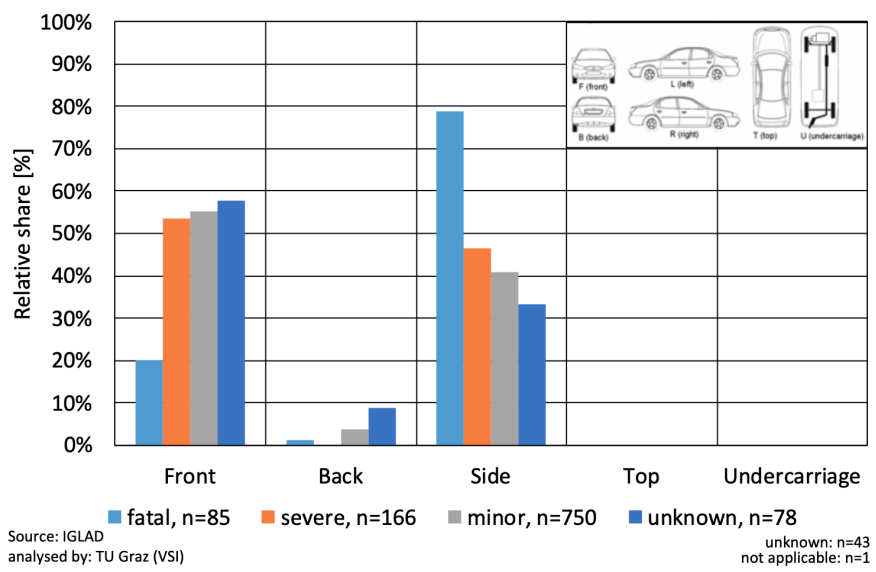

Fig. 1. General deformation area at first collision.

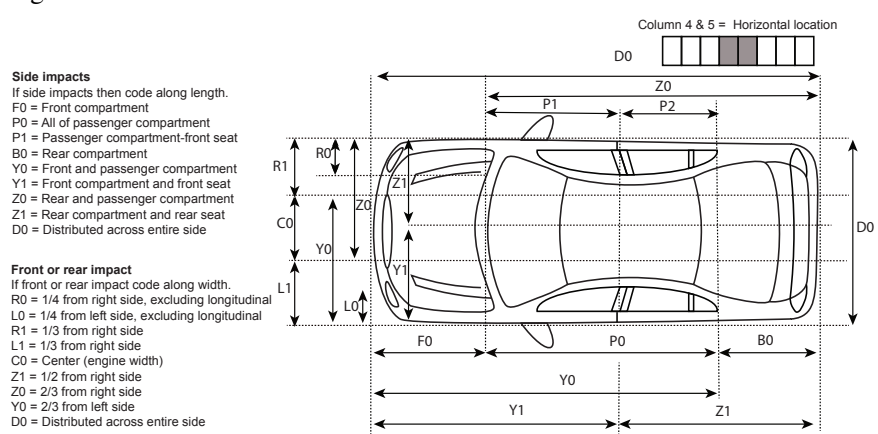

Fig. 2. Horizontal collision location classification [34].

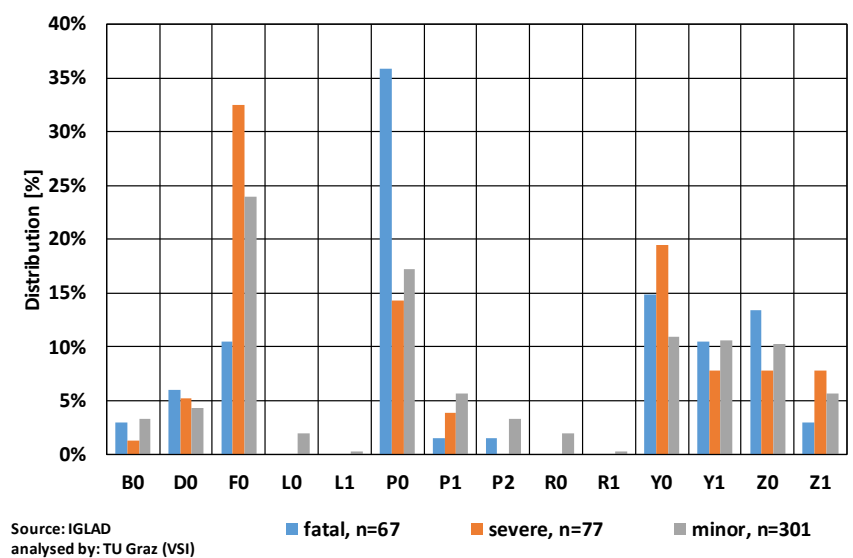

Fig. 3. Collision location of passenger cars-side impacted.

the National Highway Traffic Safety Administration (NHTSA) issued a pre-crash scenario typology that provides information on different crash types and their frequencies [36]. Based on this document, in a pre-crash scenario between two vehicles with at least one light vehicle (passenger car), the vehicle(s) turning at non-signalized junctions scenario has the second highest frequency following the lead vehicle stopping scenario. Therefore, we applied the following filter criteria to the accident data: only accidents at junctions are considered, at least one passenger car was involved in the accident, and only passenger cars against other vehicles are considered. Accidents with pedestrians, trains and unspecified participants are removed. The accident data used in this study is sourced from the Initiative for the Global harmonization of Accident Data 
Table I

RISK ESTIMATE-ODDS RATIO

\begin{tabular}{|c|c|c|c|c|c|c|c|c|}
\hline Collision Location & Description & Fatal & Severe & Minor & No Injury & Unknown & Total & $\overline{\text { ORFS }}$ \\
\hline$B_{0}$ & Rear compartment & 2 & 1 & 10 & 44 & 1 & 58 & 0.61 \\
\hline$D_{0}$ & Distributed across entire side & 4 & 4 & 13 & 5 & 1 & 27 & 1.30 \\
\hline$F_{0}$ & Front compartment & 7 & 25 & 72 & 138 & 0 & 242 & 0.91 \\
\hline$L_{0}$ & $1 / 4$ from left side & 0 & 0 & 6 & 2 & 0 & 8 & 0.0 \\
\hline$L_{1}$ & $1 / 3$ from left side & 0 & 0 & 1 & 1 & 0 & 2 & 0.0 \\
\hline$P_{0}$ & All of passenger compartment & 24 & 11 & 52 & 33 & 5 & 125 & 1.54 \\
\hline$P_{1}$ & Passenger compartment-front seat & 1 & 3 & 17 & 27 & 1 & 49 & 0.48 \\
\hline$P_{2}$ & Passenger compartment-rear seat & 1 & 0 & 10 & 11 & 0 & 22 & 0.20 \\
\hline$R_{0}$ & $1 / 4$ from right side & 0 & 0 & 6 & 5 & 0 & 11 & 0.0 \\
\hline$R_{1}$ & $1 / 3$ from right side & 0 & 0 & 1 & 1 & 0 & 2 & 0.0 \\
\hline$Y_{0}$ & Front and passenger compartment & 10 & 15 & 33 & 20 & 6 & 84 & 1.71 \\
\hline$Y_{1}$ & Front compartment and front seat & 7 & 6 & 32 & 35 & 0 & 80 & 0.83 \\
\hline$Z_{0}$ & Rear and passenger compartment & 9 & 6 & 31 & 17 & 7 & 70 & 1.01 \\
\hline$Z_{1}$ & Rear compartment and rear seat & 2 & 6 & 17 & 19 & 2 & 46 & 0.98 \\
\hline
\end{tabular}

(IGLAD) [37].

\section{B. Collision Characteristics and Classification}

Collision Deformation Classification (CDC) is a classification system defined by SAE J224 [38] that addresses the extent of damage to vehicles involved in accidents on highways. The CDC code consists of seven digits, each describing an aspect of vehicle damage. The code includes the clock direction of principal direction of force as two digits, area of deformation, specific longitudinal or lateral location of deformation, specific vertical location of deformation, type of damage distribution and maximum extent of penetration.

In addition, traffic accidents can cause fatal or severe injuries. In this study, we analyzed the accident data to establish a relationship between the impact location and the MAIS injury severity scale [15]. We initially investigated the general area of deformation (front, side and rear) against different injury severity levels (fatal, severe and minor). The results are presented in Fig. (1). The highest relative share of fatal injuries is associated with the left- and right-side impact locations. The smallest share of fatal injuries is associated with the rear of the vehicle.

To further study this relationship in detail, we investigated collisions involving side impacts separately. The longitudinal location of the vehicle body was divided into smaller parts, as shown in Fig. (2). The distribution of fatal, severe and minor injuries for a specific longitudinal impact location is visualized in Fig. (3). As seen in this graph, the complete passenger compartment $\left(P_{0}\right)$ is associated with the highest distribution of fatal injuries.

\section{Risk Estimation}

The Odds Ratio (OR) is used as a risk estimation metric to classify collisions according to their associated injury severity. OR is defined as the ratio of the probability of event $A$ occurring to the probability of event $A$ not occurring [39]. This can be estimated by the number of times the event of interest occurs divided by the number of times it does not occur. For instance, if we define the event of interest as the occurrence of fatal injuries, a nonfatal injury is equivalent to the event of interest not occurring. Odds ratio calculations within the context of this paper are presented in Appendix A through a simple example.
For each collision location, as shown in Fig. (3), the associated number of fatal, severe and minor injuries and cases of no and unknown injuries are presented in Table I. In Table I, the Odds Ratio for Fatal and Severe (ORFS) represents cases in which the event of interest is both fatal and caused severe injuries. The relationship between the impact location and injury severity for such cases is of the most interest, as we consider minor injuries acceptable in comparison. Therefore, ORFS is considered as a risk metric to rank collisions based on the impacted location. Consequently, the highest ORFS value indicates the worst collision location. The values for unknown and no injury in Table I are not considered in the OR calculations.

\section{TRAJECTORY LIBRARY}

To stay as close as possible to the original problem while maintaining an accurate model, we apply a trajectory library approach to generate feasible trajectories for the ego vehicle. These trajectories are created by solving an optimal control problem numerically.

The optimal control problem that generates the trajectory library is formulated as Eq. (1) and takes the ego vehicle from an initial position to a fine-grained grid of final positions. The cost function minimizes the Euclidean distance between the current position and the goal position at each time step and the velocity at the final position. The vehicle dynamics are included as the constrains of the optimization problem.

$$
\begin{array}{cl}
\underset{x(.), u(.)}{\operatorname{minimize}} & \int_{0}^{t_{f}}\left\|S_{g}-S(t)\right\|_{2}^{2} d t+v_{x}\left(t_{f}\right) \\
\text { subject to } & \dot{\boldsymbol{x}}(t)=f(\boldsymbol{x}(t), \boldsymbol{u}(t)), \\
& G(\boldsymbol{x}(t), \boldsymbol{u}(t)) \leq 0, \\
& \boldsymbol{x}(0)=\boldsymbol{x}_{0}, \\
& \boldsymbol{x}\left(t_{f}\right)=\boldsymbol{x}_{T} .
\end{array}
$$

In Eq. (1), $\boldsymbol{x}(t)$ is the state vector, $\boldsymbol{u}(t)$ is the control vector and $f(\boldsymbol{x}(t), \boldsymbol{u}(t))$ is a set of ordinary differential equations that represents the dynamics of the ego vehicle. The initial and final states of the vehicle are denoted as $\boldsymbol{x}_{0}$ and $\boldsymbol{x}_{T}$, respectively. The current position and the goal position of the vehicle in the global coordinate system are denoted as $S(t)$ and $S_{g}$, respectively. The path constraints of the optimization are 


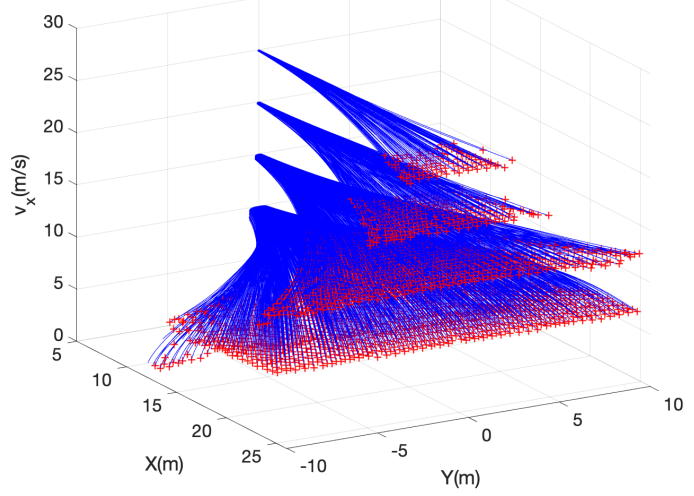

Fig. 4. Trajectory library for different initial velocities $\left(v_{x}\right) . X(t)$ and $Y(t)$ are global coordinates of center of gravity of the ego vehicle.

defined by the term $G(\boldsymbol{x}(t), \boldsymbol{u}(t))$, which contains constraints on the state and control variables of the vehicle such as limits on vehicle dynamics. Furthermore, $t_{f}$ in Eq. (1) represents the final time (defined as an optimization variable), and $v_{x}\left(t_{f}\right)$ is the longitudinal velocity of the ego vehicle at the goal position. The details of the vehicle and tire modeling are presented in Appendix B.

The optimization problem is solved with the CasADi Opti functionality [40]. By using the direct multiple shooting method [41], the optimal control problem in Eq. (1) is discretized in time and transformed into a Nonlinear Programming Problem (NLP). The resulting NLP problem is then solved numerically by the interior point method with IPOPT [42]. Furthermore, the direct multiple shooting is capable of dealing with strong nonlinear optimization problems [43] and is cost efficient in terms of computation time [44].

Therefore, a trajectory library is created for a range of initial velocities. All trajectories are derived by combining braking and steering, enforced as a hard optimization constraint through the term $G(\boldsymbol{x}(t), \boldsymbol{u}(t))$ in Eq. (1). During the run-time operation of the ego vehicle, a subset of trajectories from the library is chosen based on the vehicle's current velocity. An example of this trajectory library for different initial velocities is presented in Fig. (4), with the red markers representing the reachable goal positions for the vehicle.

\section{Collision Severity Minimization Motion PLANNING}

Thus far, we have introduced a metric that maps collision severity and impact location and generated a trajectory library. In this section, we present how this metric is used in the cost function of a motion planning framework. We also introduce our assumptions and our approach to detecting a collision and determining the type of collision.

\section{A. Assumptions}

To predict the future trajectories of surrounding vehicles, a kinematic bicycle model [45] is applied to the current state of the vehicle. We assume that this current state is exactly known and that the prediction model provides an accurate representation of the movement of the vehicle. Even though the presence of uncertainties may affect the resulting predictions, characteristics of collision scenarios such as a short prediction horizon and close proximity of vehicles to each other make the application of a physics-based motion model for trajectory prediction reasonable [46], [47]. The position, velocity and heading angle of the vehicles at the time of prediction is determined by the ego vehicle's perception system. Furthermore, this study is performed in the absence of large time delays, e.g., sensor or actuator delays. The evaluated scenarios are junctions and are encoded in the vehicle's system beforehand. It is also assumed that the ego vehicle is fully automated, but other vehicles can be manual or with lower levels of automation. No vehicle-to-vehicle communication is present.

\section{B. Trajectory Selection Cost Function}

The overall goal of the cost function prior to an imminent unavoidable collision is to minimize post-impact consequences in terms of injury severity for occupants. The cost function that defines the criteria for trajectory selection is formulated as follows:

$$
J=\underbrace{W_{1} \times V_{r e l_{n}}\left(t_{c}\right)}_{J_{0}}+F\left(P_{n}\left(t_{c}\right)\right),
$$

where $t_{c}$ is the instant of time that a collision between the ego vehicle and another vehicle is determined. $V_{\text {rel }_{n}}$ is the relative velocity between the ego vehicle and the other vehicle(defined in Eq. (C.1) in Appendix C), and subscript $n$ refers to the $n^{\text {th }}$ trajectory of the ego vehicle in the library. The term $F\left(P_{n}\left(t_{c}\right)\right)$ outputs the cost based on the impact location, and $P_{n}\left(t_{c}\right)$ is an abstract term that represents the calculations performed to determine the impacted location at the time of $t_{c}$. The cost function $F\left(P_{n}\left(t_{c}\right)\right)$ is derived based on ORFS values that are presented in Table I and the analysis of Fig. (1). The side impact location with the highest ORFS is assigned the highest cost value, and the side impact location with the lowest ORFS is assigned the lowest cost value. We assign values from 1-12 to $F\left(P_{n}\left(t_{c}\right)\right)$ as presented in Eq. (3); these are merely categorical.

$$
\begin{cases}12 & \text { front and passenger compartment }\left(Y_{0}\right) \\ 11 & \text { all of passenger compartment }\left(P_{0}\right) \\ 10 & \text { distributed across entire side }\left(D_{0}\right) \\ 9 & \text { rear and passenger compartment }\left(Z_{0}\right) \\ 8 & \text { rear compartment and rear seat }\left(Z_{1}\right) \\ 7 & \text { front compartment }\left(F_{0}\right) \\ 6 & \text { front compartment and front seat }\left(Y_{1}\right) \\ 5 & \text { rear compartment }\left(B_{0}\right) \\ 4 & \text { passenger compartment-front seat }\left(P_{1}\right) \\ 3 & \text { passenger compartment-rear seat }\left(P_{2}\right) \\ 2 & \text { front to front collision } \\ 1 & \text { front to rear collision }\end{cases}
$$

Since our aim is to investigate the effect of the impact location on injury severity, we consider this collision severity factor as the dominant one in the cost function. To further reduce the collision severity, the velocity term $V_{\text {rel }}$ is added 
to the cost function. This term is multiplied by a weighting factor of $W_{1}$ leading to the value of $J_{0}$ being less than 1 . With this approach, a trajectory with a lower cost of impact location always has priority over a trajectory with a higher cost of impact location. Moreover, for trajectories with the same impact location, a trajectory with lower $V_{r e l}$ has priority over a trajectory with a higher $V_{\text {rel }}$.

\section{Collision Detection}

Collision detection at each time step is performed through the concept of robotic occupancy [48]. The surrounding environment of the ego vehicle is divided into free and occupied spaces and is represented as discrete grids. At each time step all cells overlapped by the rectangle that approximates the position of the other vehicle are defined as occupied. The area outside the road is also defined as occupied. A collision is determined if any points on the rectangle that represents the body of the ego vehicle belong to occupied spaces. These points are determined by calculating the vertices of the ego vehicle rectangle and dividing the space between each pair of points into a set of points.

\section{Type of Collision}

To calculate the cost of collision according to Eq. (3), we propose a method to determine the impact location in a collision between two arbitrary vehicles. Our approach starts by dividing the rectangle that represents the body of the impacted vehicle into ten polygons and labeling them from 1 to 10 as shown in Fig. (5). Polygons 10 and 5 represent the front and rear of the vehicle, respectively. The right and left sides of the vehicle are each divided into four polygons (1-4 and 6-9). Furthermore, polygons labeled as 1 and 9 are associated with $F_{0}$ impact location, 2 and 8 with $P_{1}, 3$ and 7 with $P_{2}$, and 4 and 6 with $B_{0}$. An overlapping impact location is derived from a combination of polygons. For instance, Polygons 2 and 3 together construct a $P_{0}$ collision location.

Then, we calculate a set of points on the rectangle that represents the body of the impact vehicle. This calculation is performed similarly to the method discussed in Section VI.B. The collision location is thus determined by identifying the polygon or polygons of the impacted vehicle to which these points belong. Therefore, by identifying the polygon number, the collision location is determined and a cost is allocated based on Eq. (3). This is further illustrated through a collision between the ego vehicle (impact vehicle) and another vehicle (impacted vehicle) in Fig. (6). In this example, points (shown with black markers) on the rectangle of the ego vehicle (red) belong to Polygon 8 of the other vehicle (blue). Thus, the collision location is the front seat $\left(P_{1}\right)$ of the other vehicle, and a cost is allocated according to Eq. (3).

Furthermore, since the severity is determined by the collision location on the vehicle that is impacted, it is required to determine which one of the two vehicles is the impacted one. Consequently, in order to identify the impacted vehicle and location the rectangle of each vehicle is divided into ten polygons and sets of points are calculated for both vehicles.

This is followed by defining Polygon 10, i.e., front of vehicle, as the impact polygon. If any points on the body of the other

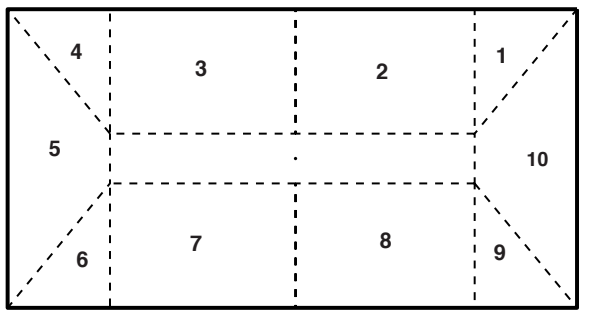

Fig. 5. Vehicle body that is divided into ten polygons.

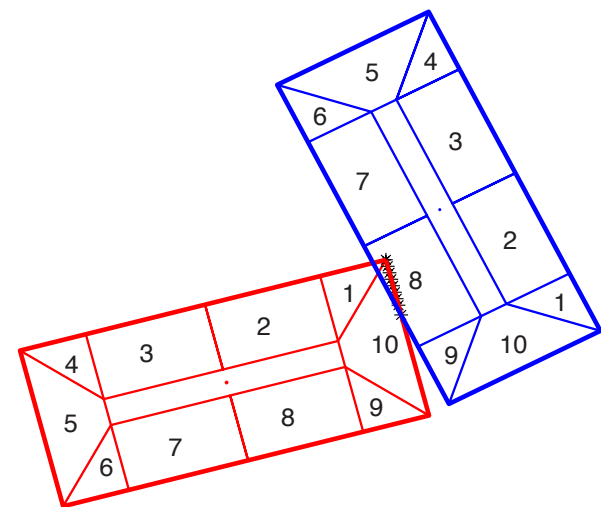

Fig. 6. Example of $P_{1}$ side collision between the ego vehicle (red) and the other vehicle (blue).

vehicle belong to Polygon 10 of the ego vehicle, then the front of the ego vehicle is involved in the collision, i.e., the ego vehicle impacts the other vehicle. We define this as a primary type of collision. Similarly, if any points on the ego vehicle belong to Polygon 10 of the other vehicle, then the front of the other vehicle is involved in the collision, i.e., the ego vehicle is impacted. We call this a secondary type of collision. If only Polygon 10 of both vehicles intersect with each other, then the collision is considered as a front-to-front collision with the associated cost value as per Eq. (3).

In a primary type of collision, the polygon number of the other vehicle determines the collision location and the associated cost. On the other hand, in a secondary type of collision, the impacted polygon on the ego vehicle determines the collision location and cost. For instance, in Fig. (6), Polygon 10 (front) of the ego vehicle is involved in the collision, and the type of collision is primary; Polygon 8 of the other vehicle is the collision location and determines the collision cost.

\section{RESULTS}

To demonstrate that our method is able to minimize collision severity, two scenarios are simulated and analyzed. The first scenario is a T-junction and involves two vehicles (ego and another vehicle). However, as the number of interacting traffic agents increases, risk estimation, in terms of which type of collision is more severe, and decision-making, in terms of which trajectory to follow, become more complex. Therefore, the second scenario presents a case involving multiple vehicles.

\section{A. Two-Vehicle Scenario}

The scenario involves an ego vehicle, another passenger car and a T-shaped junction. A general description of the scenario is presented in Fig. (7). The direction of movement for the 


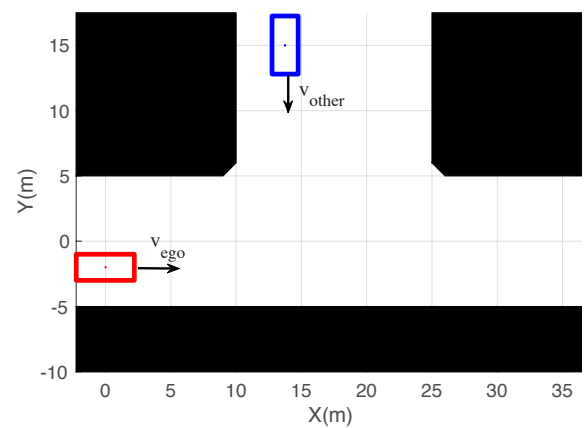

Fig. 7. General description of the scenario and positions of the ego vehicle (red) and the other vehicle (blue) at $t=0 \mathrm{~s}$. Initial velocity of both vehicles is $v=15 \mathrm{~m} / \mathrm{s}$ in arrow direction.

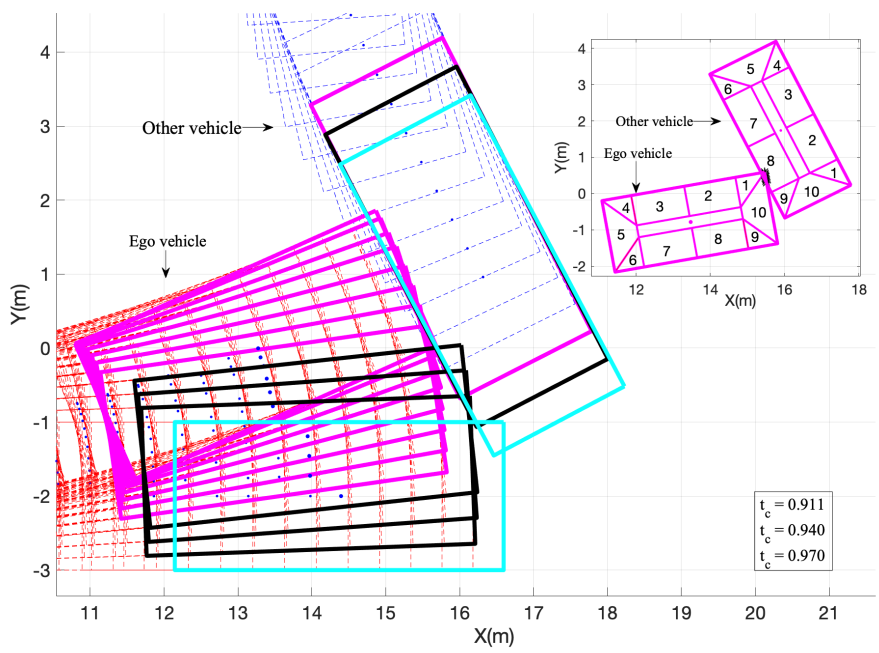

Fig. 8. Collisions between different trajectories of the ego vehicle and the other vehicle at different time steps.

Table II

COLLISION COST

\begin{tabular}{ccc}
\hline \hline Predicted Collision Time & Trajectory No. & Cost \\
\hline $0.911(\mathrm{~s})$ & $159-162$ & $4 \leq \operatorname{cost}<5$ \\
$0.911(\mathrm{~s})$ & $163-165$ & $11 \leq \cos t<12$ \\
$0.911(\mathrm{~s})$ & 166 & cost $\geq 12$ \\
$0.940(\mathrm{~s})$ & 156 & $7 \leq \cos t<8$ \\
$0.940(\mathrm{~s})$ & $157-158$ & $6 \leq \operatorname{cost}<7$ \\
$0.970(\mathrm{~s})$ & 155 & $7 \leq \cos t<8$ \\
\hline
\end{tabular}

ego vehicle and the other vehicle is from left to right and top to bottom, respectively. The velocity of both vehicles (ego and other) is $15 \mathrm{~m} / \mathrm{s}$ at $t=0$, and the trajectory of the other vehicle is predicted for approximately the next $1 s$. A subset of trajectories for the ego vehicle is selected from the trajectory library based on its current velocity. The prediction horizon is divided into time steps, and at each time step a collision check is performed as outlined in Section VI.B.

The predicted collisions between different trajectories of the ego vehicle and the other vehicle at various time steps are presented in Fig. (8). The initial collision is determined at $t_{c}=0.911 \mathrm{~s}$, where a subset of trajectories of the ego vehicle, namely, trajectory numbers $159-166$, collide with the other vehicle. Colliding trajectories at this time step are presented with magenta boxes for both vehicles. One of the trajectories at this time step, trajectory number 160 , is shown in more

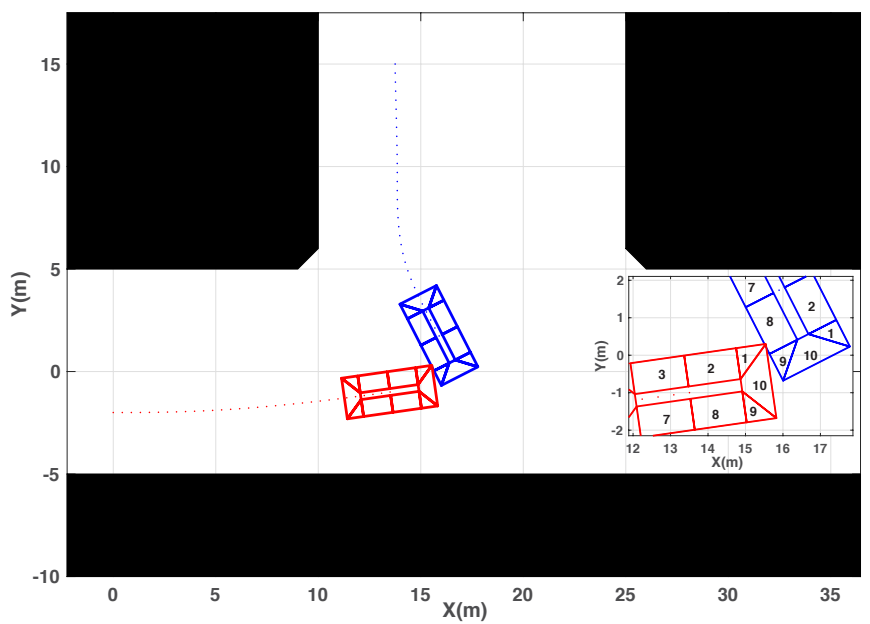

Fig. 9. Optimal trajectory of the ego vehicle (red) leading to minimum collision severity and the path of the other vehicle (blue) until $t_{c}=0.911 \mathrm{~s}$.

detail in the top right corner of Fig. (8). As we can see in this graph and for this trajectory, points from Polygon 10 of the ego vehicle are inside Polygon 8 of the other vehicle. This indicates that the ego vehicle strikes the other vehicle, and the front seat of the passenger compartment $(P 1)$ is the collision location on the other vehicle. Thus, the associated cost for this trajectory before considering the effects of velocity is 4 (see Eq. (3)). The trajectories leading to collisions at the current time step are then removed from the library in order to not be considered as the cause of collision in subsequent time steps. They are instead added to the collision set with their associated costs. The collision cost for all trajectories at this time step is presented in Table II.

The next time step (predicted collision) is $t_{c}=0.940 \mathrm{~s}$, and the intersecting rectangles are shown in black in Fig. (8). The colliding trajectories at this time step are labeled as $156-158$. Collisions at this time step are also determined as primary, and the associated cost is presented in Table (II). For instance, trajectory 157 has a cost value of 6 , meaning that both the front compartment $\left(F_{0}\right)$ and front seat $\left(P_{1}\right)$ of the other vehicle are impacted by the front of the ego vehicle, and the collision location is $Y_{1}$. This location was determined by identifying a set of points on Polygon 10 of the ego vehicle that belonged to both Polygon 8 and 9 of the other vehicle.

Finally, at $t_{c}=0.970 \mathrm{~s}$, the last remaining trajectory in the set leads to a side impact, where the impact location is the front compartment $\left(F_{0}\right)$. The colliding rectangles at this time step are presented in cyan. All trajectories and their associated costs at various time steps are summarized in Table II.

As presented in Table II, the lowest cost trajectories are 159-162 and are associated with the $P_{1}$ impact location. Since these trajectories have the same impact location, the trajectory with the lowest relative velocity is chosen for execution. In cases where several trajectories have the same cost, the first one in the set is selected for the ego vehicle to follow. In this example scenario, trajectory 159 has the lowest cost (4.114). Since no collision-free trajectory exists in the selected subset, it is optimal to follow this trajectory in terms of minimizing the injury severity of vehicle passengers. 

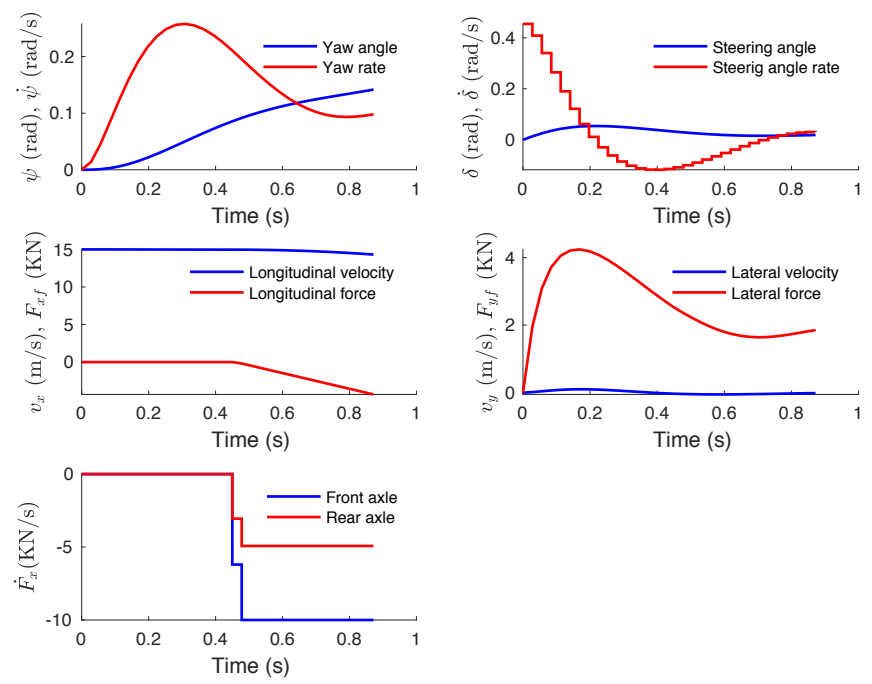

Fig. 10. State variables and control inputs of the ego vehicle to follow the optimal trajectory (trajectory 159$)$ until just before the collision $\left(t_{c}=0.911 \mathrm{~s}\right)$.

The trajectory of the ego vehicle with the minimum cost, as well as the path of the other vehicle, is presented in Fig. (9) from $t=0$ until the time of collision. The vehicle boxes at the point of collision are also enlarged in this figure. As shown, Polygon 10 of the ego vehicle intersects with Polygon 8 of the other vehicle. Therefore, this collision is categorized as $P 1$ with a cost of 4 .

The state variables including heading and steering angle, yaw rate, longitudinal and lateral velocity and longitudinal and lateral tire forces for the selected optimal trajectory are presented in Fig. (10). For the ego vehicle to follow the minimum collision severity trajectory, the control inputs (steering angle rate and rate of change of braking) until the collision time are also as demonstrated in Fig. (10).

\section{B. Multi-Vehicle Scenario}

The collision severity minimization motion planning approach can be extended to handle scenarios involving more than one vehicle in the surrounding environment of the ego vehicle. An example scenario is an intersection with the ego vehicle and two other vehicles (I and II). The velocity of ego vehicle at $t=0$ is $v=20 \mathrm{~m} / \mathrm{s}$ and for other vehicles is $v=15 \mathrm{~m} / \mathrm{s}$. This scenario at the time of collision is shown in Fig. (11) and Fig. (12). These figures present collisions at different time steps in a single scenario, but for the sake of visibility, the former shows collisions with one vehicle (I) and the latter collisions with another (II). All collisions with vehicle (I) are determined as the secondary type since the ego vehicle is impacted by the other vehicle (I). On the other hand, all collisions with vehicle (II) are of the primary type (see Section VI.D). The first collision is determined at $t_{c}=0.853 \mathrm{~s}$ between the ego vehicle and the other vehicle (I), and the colliding trajectories are presented with black boxes in Fig. (11). The collision at this time for trajectory 291 is presented in more detail in the top right corner of this figure. As we can see, two polygons (2 and 3 ) of the ego vehicle are impacted, meaning that the collision location is $P_{0}$; furthermore, and according to Eq. (3), the cost is 11 .

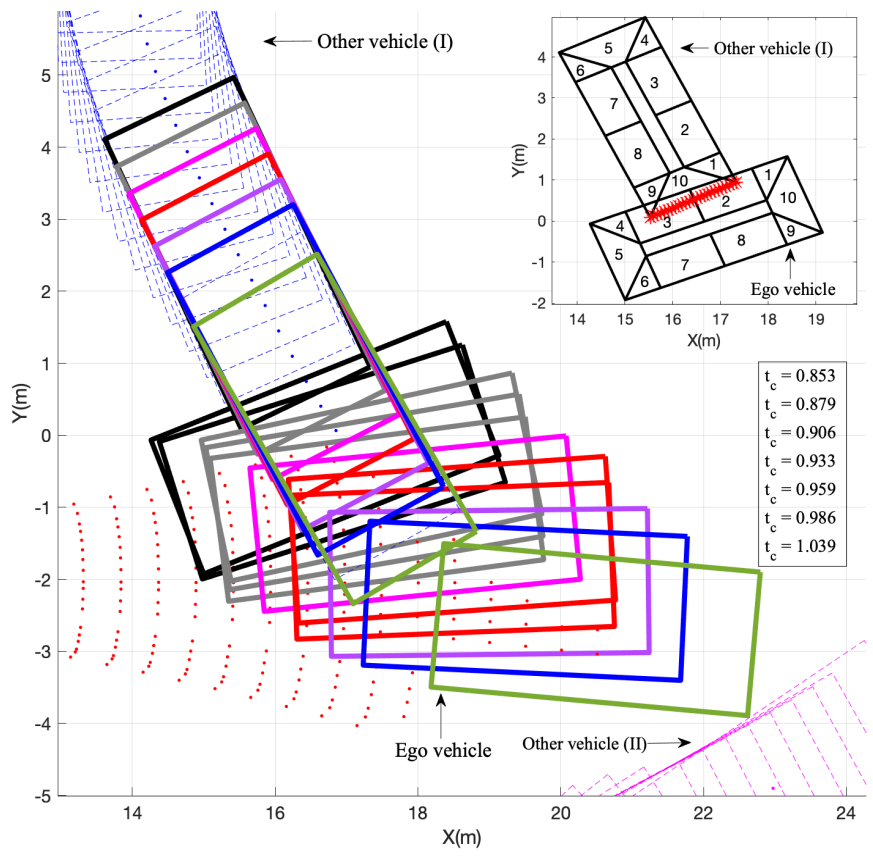

Fig. 11. Collisions of the ego vehicle with the other vehicle (I) at different time steps.

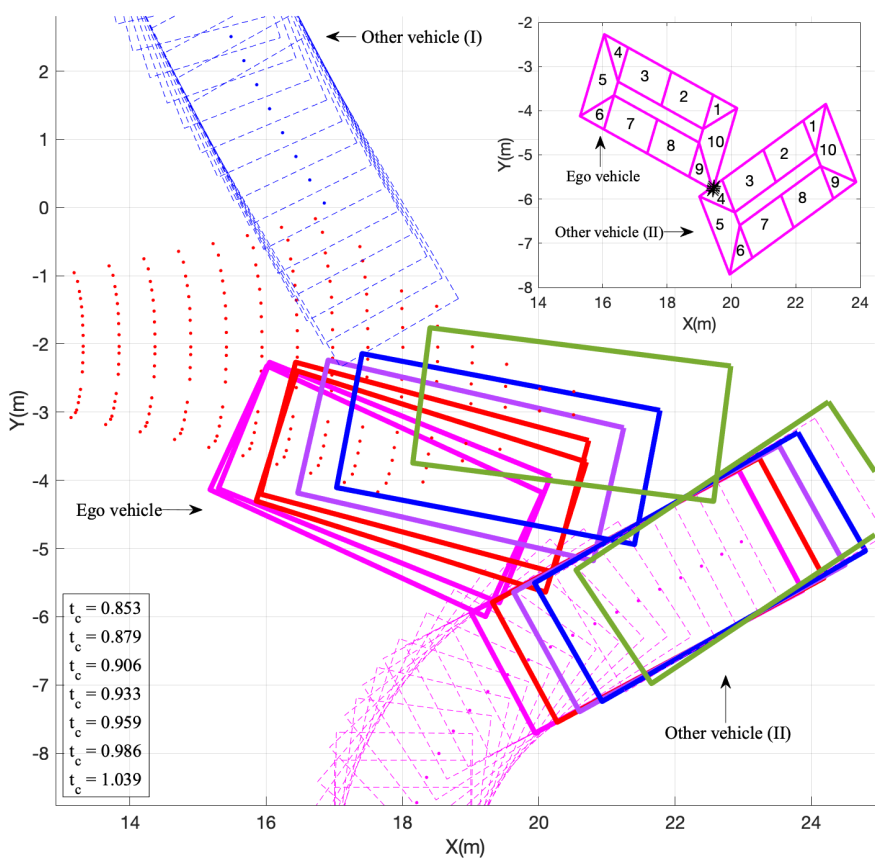

Fig. 12. Collisions of the ego vehicle with the other vehicle (II) at different time steps.

The first collision between the ego vehicle and the other vehicle (II) is predicted at $t_{c}=0.906 \mathrm{~s}$. One of the colliding trajectories (293) is shown in more detail in the top right corner of this figure. For this trajectory, the rear compartment $B_{0}$ of vehicle (II) is impacted by the ego vehicle, and the cost is 5 . Trajectory 304 of the ego vehicle is also involved in a collision at this time step but with vehicle (I). The colliding boxes are shown in magenta in Fig. (11) and Fig. (12).

From $t_{c}=0.906 \mathrm{~s}$ and onward, the ego vehicle is involved in collisions with both vehicles (I and II). For instance, at $t_{c}=0.933 \mathrm{~s}$, two of the trajectories of the ego vehicle $(302$ 


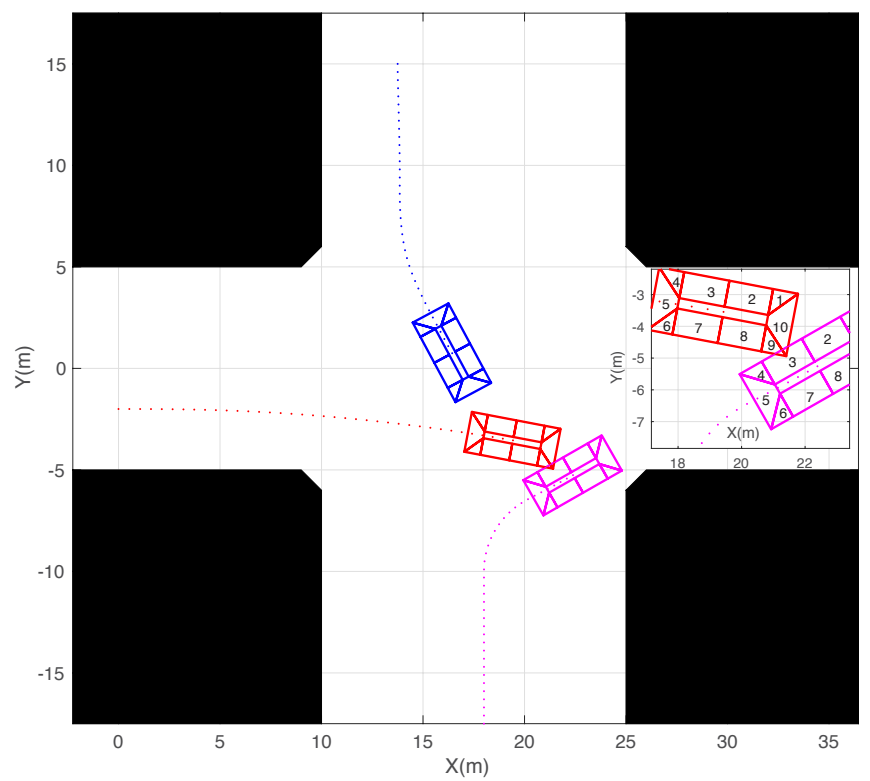

Fig. 13. Optimal trajectory (297) of the ego vehicle (red) leading to a collision with minimum severity with the other vehicle (II) (magenta). The path of the other vehicle (I) (blue) is also shown.
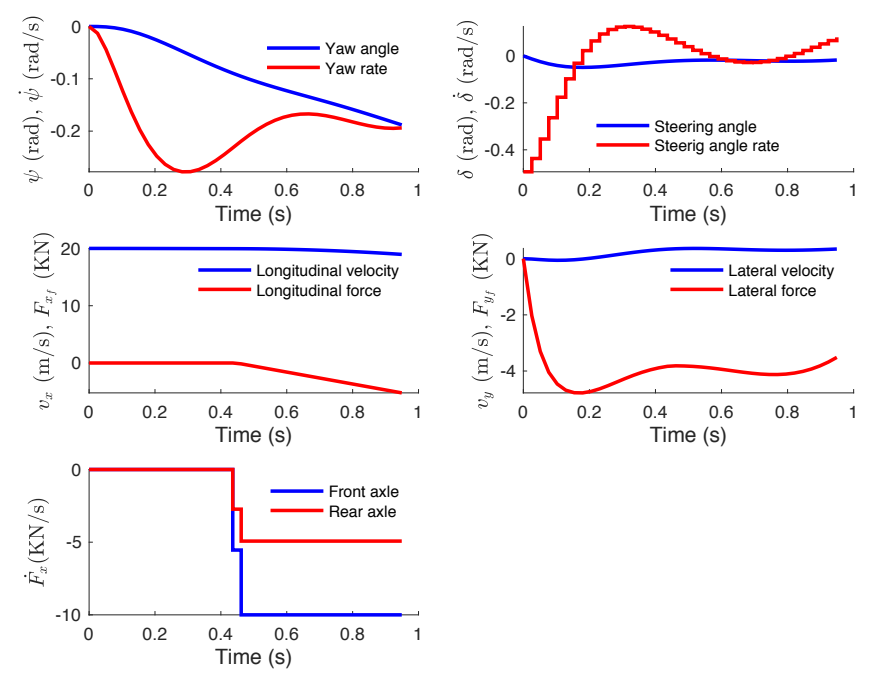

Fig. 14. State variables and control inputs of the ego vehicle to follow the optimal trajectory (297) until just before the collision $\left(t_{c}=0.986 s\right)$.

303) are involved in a collision with vehicle (I) and the other two (294-295) with vehicle (II). These collisions are presented with red boxes in Fig. (11) and Fig. (12).

The colliding trajectories from the set and their associated costs are summarized in Table (III) and Table (IV). The trajectory with the lowest collision severity is determined exactly as in scenarios involving only one other vehicle and is presented in Fig. (13). This trajectory is labeled as 297 and leads to a $P_{2}$ collision with the other vehicle (II) at $t_{c}=0.986 \mathrm{~s}$. The state and control inputs for the chosen trajectory, leading to the minimum collision severity, are presented in Fig. (14).

\section{DISCUSSION}

The scenarios presented in the results successfully demonstrate the application of collision severity minimization in motion planning for unavoidable collision situations. Since
Table III

COLLISION COST FOR COLLISIONS OF EGO VEHICLE AND VEHICLE (I)

\begin{tabular}{ccc}
\hline \hline Predicted Collision Time (s) & Trajectory No. & Cost \\
\hline 0.853 & 291 and 308 & $11 \leq \operatorname{cost}<12$ \\
0.879 & 305 & $5 \leq \operatorname{cost}<6$ \\
0.879 & $306-307$ & $8 \leq \operatorname{cost}<9$ \\
0.906 & 304 & $5 \leq \operatorname{cost}<6$ \\
0.933 & $302-303$ & $5 \leq \cos t<6$ \\
0.959 & 301 & $5 \leq \cos t<6$ \\
0.986 & 300 & $5 \leq \cos t<6$ \\
1.039 & 299 & $5 \leq \cos t<6$ \\
\hline
\end{tabular}

Table IV

COLLISION COST FOR COLLISIONS OF EGO VEHICLE AND VEHICLE (II)

\begin{tabular}{ccc}
\hline \hline Predicted Collision Time (s) & Trajectory No. & Cost \\
\hline 0.906 & $292-293$ & $5 \leq \cos t<6$ \\
0.933 & 294 & $8 \leq \cos t<9$ \\
0.933 & 295 & $3 \leq \cos t<4$ \\
0.959 & 296 & $3 \leq \cos t<4$ \\
0.986 & 297 & $3 \leq \cos t<4$ \\
1.039 & 298 & $5 \leq \cos t<6$ \\
\hline
\end{tabular}

approximately $43 \%$ of the accidents in Europe occur at intersections [49], the demonstrated method is thus promising in many collision situations involving road vehicles. The presented method can also be extended to more complex scenarios. However, the limiting factor, especially in the latter case, is most likely not the method but the data that is driving the choice of impact location.

A relatively straightforward decision between side locations, as shown in the results, is supported both by existing data and logic. However, there is a shortage of data on the severity of collisions related to the heterogeneity of vehicle design. This lack of data could make it difficult to choose between trajectories in scenarios where more than two vehicles are involved in a collision (see Section VII.B). For example, in the scenario of Fig. (13), the mass and height of the involved vehicles could affect the choice of the optimal impact location. Such aspects can easily be included in the suggested method by using expert models such as [10], but this risks aggravating problems with model complexity. Indeed, the heterogeneity of vehicle design will thus make it imperative for the suggested method that future vehicles are fitted with sensors that allow for post-collision statistical analysis. Fortunately, highly automated vehicles will most likely not only be required to generate such data, but vehicle manufacturers will also be required to share this data across the automotive industry. Therefore, the proposed method is in line with the evolution of the automotive industry.

There are other similar opportunities for improving the suggested method. For the proposed implementation, the $X$ and $Y$ coordinates of the goal positions have a distance of $0.5 \mathrm{~m}$. A finer grid of goal positions increases the number of trajectories in the set. Consequently, a lower cost trajectory might be identified in certain scenarios. However, there is a trade-off between identifying the lowest cost trajectory and the number of trajectories that can be checked before an imminent collision. Thus, a better trajectory might still not be chosen from the trajectory library, as it may not be possible to check all trajectories in time to act appropriately before a collision 
occurs.

The post-impact behavior of the vehicles has not yet been explored. What occurs after the collision may influence the final cost and thus the minimum cost trajectory. For example, in the scenario of Fig. (13) depending on the positions and velocities of the ego vehicle and other vehicle (II) after the initial impact, a secondary collision may occur between any of the vehicles involved in the scenario.

Similarly, several vehicles can be involved in a simultaneous collision. This situation can be handled by the suggested approach by considering each collision in isolation and allocating a collision cost according to a one-to-one vehicle collision. However, it is only an assumption that the presented data is comprehensive enough to build relationships and draw conclusions in this case. Therefore, addressing this type of collision might be better postponed to when more data on simultaneous collisions become available.

\section{COnclusion And Future Work}

We proposed a motion planning method that specifically addresses the minimization of collision severity in unavoidable collision situations through a trajectory library containing reference trajectories. The results of the analysis of real accident data on impact location and injury severity allowed us to build realistic criteria to rank trajectories from the most severe to the least severe. The concept of a trajectory library allowed us to incorporate a nonlinear vehicle and tire model to stay closer to the original problem, as defined in the problem statement. Simulations verified that the proposed method is able to mitigate the severity of a collision in different scenarios.

The suggested method shows a clear promise in regard to extending the cost function by integrating more collision factors as well as including the post-impact motion of vehicles in decision-making. Potential extensions of our method could consider uncertainty in problem formulation, both in terms of trajectory prediction and driver intention. The extensions of the method could also incorporate what happens after the first impact in terms of vehicles final positions and explore the possibility of secondary collisions or collisions with other road users.

\section{APPENDIX A \\ ODDS RATIO CALCULATIONS}

We define the occurrence of fatal and severe injuries as the event of interest, and observations of minor injuries equivalent to the event of interest not occurring. To estimate the risk of fatal and severe injuries for a specific impact location, e.g., $P_{0}$ in Table A.1, we apply the formula in Eq. (A.1), where $a$ is the sum of fatal and severe injuries for $P_{0}$ and $b$ represents the minor ones. Similarly, $c$ is the sum of all observed fatal and severe injuries for all other impact locations excluding $P_{0}$ and $d$ is the sum of all minor injuries for all other impact locations.

$$
O R=\frac{a / b}{c / d}
$$

The odds of being seriously injured (fatal and severe) for one impact location divided by the odds of being seriously injured for all other locations is the metric for risk estimation.
Table A.1

ODDS RATIO

\begin{tabular}{ccc}
\hline \hline Collision location & Fatal and severe & Minor \\
\hline$P_{0}$ & $a$ & $b$ \\
All other locations & $c$ & $d$ \\
\hline
\end{tabular}

\section{APPENDIX B}

VeHicle AND TIRE MODELING

The motion of the ego vehicle is modeled as a dynamic bicycle model. Braking and steering are controlled through the rate of change of longitudinal tire forces and the rate of change of the steering angle. The model is sufficient for highly dynamic scenarios. The system dynamics are described as follows:

$$
\begin{aligned}
& \dot{X}=v_{x} \cos (\psi)-v_{y} \sin (\psi), \\
& \dot{Y}=v_{x} \sin (\psi)+v_{y} \cos (\psi), \\
& \dot{\psi}=r \\
& \dot{v}_{x}=\frac{1}{m}\left(F_{x_{f}} \cos (\delta)+F_{x_{r}}-F_{y_{f}} \sin (\delta)\right)+\dot{\psi} v_{y}, \\
& \dot{v}_{y}=\frac{1}{m}\left(F_{x_{f}} \sin (\delta)+F_{y_{r}}+F_{y_{f}} \cos (\delta)\right)-\dot{\psi} v_{x}, \\
& \left.\dot{r}=\frac{1}{I_{z}}\left(L_{f} F_{x_{f}} \sin (\delta)+L_{f} F_{y_{f}} \cos (\delta)\right)-L r F_{y_{r}}\right), \\
& \dot{\delta}=u_{1}, \\
& \dot{F}_{x_{f}}=u_{2}, \\
& \dot{F}_{x_{r}}=u_{3},
\end{aligned}
$$

where $X$ and $Y$ are the position of the center of gravity of the vehicle in global coordinates, $\psi$ is the heading angle of the vehicle body, and $v_{x}, v_{y}$ and $r$ are the longitudinal, lateral, and rotational velocity of the vehicle body, respectively. The steering angle is denoted by $\delta$, and longitudinal and lateral forces on the front and rear axles are $F_{x_{f}}$ and $F_{x_{r}}$, respectively. The control inputs are as follows: rate of change of steering angle $\left(u_{1}\right)$, and rate of change of longitudinal tire forces for both front $\left(u_{2}\right)$ and rear $\left(u_{3}\right)$ axles. The vehicle parameters and their values are defined in Table B.1.

A simplified version of the nonlinear Pacejka Magic Formula tire model is used in this paper based on the work done by [50]. The lateral tire forces on the front and rear axles are according to Eq. (B.2). In the following equations, $f$ and $r$ indices refer to the front and rear wheels, respectively.

$$
\begin{aligned}
& F_{y_{f}}=-\sin \left(\tan ^{-1}\left(C_{f} \alpha_{f}\right)\right) \sqrt{\left(\mu F_{z_{f}}\right)^{2}-F_{x_{f}}^{2}}, \\
& F_{y_{r}}=-\sin \left(\tan ^{-1}\left(C_{r} \alpha_{r}\right)\right) \sqrt{\left(\mu F_{z_{r}}\right)^{2}-F_{x_{r}}^{2}} .
\end{aligned}
$$

In Eq. (B.2), $C$ represents the wheel tire stiffness. The slip angles for the front and rear axles are defined as

$$
\begin{aligned}
& \alpha_{f}=\tan ^{-1}\left(\frac{v_{y}+L_{f} \dot{\psi}}{\left|v_{x}\right|}\right)-\delta, \\
& \alpha_{r}=\tan ^{-1}\left(\frac{v_{y}-L_{r} \dot{\psi}}{\left|v_{x}\right|}\right) .
\end{aligned}
$$

The load transfer between axles is also considered, and the normal load on the front and rear axles are calculated as follows:

$$
\begin{aligned}
& F_{z_{f}}=\left(m g L_{r}-h_{C G} m a_{x}\right) / L_{f}+L_{r}, \\
& F_{z_{r}}=\left(m g L_{f}+h_{C G} m a_{x}\right) / L_{f}+L_{r},
\end{aligned}
$$


Table B.1

Vehicle Parameters of the Ego Vehicle

\begin{tabular}{lll}
\hline \hline Parameter & Description & Value \\
\hline$m$ & Mass of ego vehicle & $2150 \mathrm{~kg}$ \\
$I_{z}$ & Moment of Inertia & $3075 \mathrm{kgm}^{2}$ \\
$L_{f}$ & Distance between front axle and center of gravity & $1.4 \mathrm{~m}$ \\
$L_{r}$ & Distance between rear axle and center of gravity & $1.45 \mathrm{~m}$ \\
$h$ & Center of gravity to ground height & $0.738 \mathrm{~m}$ \\
$C_{f}$ & Front wheel tire stiffness & 22 \\
$C_{r}$ & Rear wheel tire stiffness & 22 \\
$\mu$ & Friction coefficient & 0.763 \\
\hline
\end{tabular}

where acceleration $a_{x}$ is defined according to Eq. (B.5).

$$
a_{x}=\left(F_{x_{f}}+F_{x_{r}}\right) / m
$$

\section{APPENDIX C \\ RELATIVE VELOCITY}

The relative velocity $\left(V_{\text {rel }}\right)$ from Eq. (2) is calculated as shown in Eq. (C.1), where the subscripts $e$ and $o$ refer to the ego vehicle and the other vehicle, respectively. The heading angle and velocity of the other vehicle are denoted as $\phi$ and $v_{o}$, respectively. $X$ and $Y$ represent the global directions and $V$ is the velocity in these directions. Definitions of variables for the ego vehicle were previously defined in Appendix B.

$$
\begin{aligned}
& V_{e_{X}}=v_{x} \cos (\psi)-v_{y} \sin (\psi), V_{o_{X}}=v_{o} \cos (\phi), \\
& V_{e_{Y}}=v_{x} \sin (\psi)+v_{y} \cos (\psi), V_{o_{Y}}=v_{o} \sin (\phi), \\
& V_{r e l_{X}}=V_{o_{X}}-V_{e_{X}}, V_{r e l_{Y}}=V_{o_{Y}}-V_{e_{Y}}, \\
& V_{\text {rel }}=\sqrt{\left(V_{r e l_{X}}\right)^{2}+\left(V_{r e l_{Y}}\right)^{2}}
\end{aligned}
$$

\section{REFERENCES}

[1] T. Luettel, M. Himmelsbach, and H. Wuensche, "Autonomous ground vehicles-concepts and a path to the future," Proceedings of the IEEE, vol. 100, no. Special Centennial Issue, pp. 1831-1839, May 2012.

[2] L. Svensson, L. Masson, N. Mohan, E. Ward, A. P. Brenden, L. Feng, and M. Törngren, "Safe stop trajectory planning for highly automated vehicles: An optimal control problem formulation," in 2018 IEEE Intelligent Vehicles Symposium (IV), June 2018, pp. 517-522.

[3] J. Salvado, L. M. M. Custódio, and D. Hess, "Contingency planning for automated vehicles," in 2016 IEEE/RSJ International Conference on Intelligent Robots and Systems (IROS), Oct 2016, pp. 2853-2858.

[4] K. Lee and D. Kum, "Collision avoidance/mitigation system: Motion planning of autonomous vehicle via predictive occupancy map," IEEE Access, vol. 7, pp. 52 846-52 857, 2019.

[5] S. Magdici and M. Althoff, "Fail-safe motion planning of autonomous vehicles," in 2016 IEEE 19th International Conference on Intelligent Transportation Systems (ITSC), Nov 2016, pp. 452-458.

[6] J. P. Alsterda, M. Brown, and J. C. Gerdes, "Contingency model predictive control for automated vehicles," 2019 American Control Conference (ACC), pp. 717-722, 2019.

[7] J. Hardy and M. Campbell, "Contingency planning over probabilistic obstacle predictions for autonomous road vehicles," IEEE Transactions on Robotics, vol. 29, no. 4, pp. 913-929, Aug 2013.

[8] C. Katrakazas, M. Quddus, W.-H. Chen, and L. Deka, "Real-time motion planning methods for autonomous on-road driving: State-of-the-art and future research directions," Transportation Research Part C: Emerging Technologies, vol. 60, pp. 416 - 442, 2015.

[9] F. M. Favaró, N. Nader, S. O. Eurich, M. Tripp, and N. Varadaraju, "Examining accident reports involving autonomous vehicles in california," PLOS ONE, vol. 12, no. 9, pp. 1-20, 09 2017. [Online]. Available: https://doi.org/10.1371/journal.pone.0184952

[10] H. Wang, Y. Huang, A. Khajepour, Y. Zhang, Y. Rasekhipour, and D. Cao, "Crash mitigation in motion planning for autonomous vehicles," IEEE Transactions on Intelligent Transportation Systems, vol. 20, no. 9, pp. 3313-3323, Sep. 2019.

[11] B. Simon, F. Franke, P. Riegl, and A. Gaull, "Motion planning for collision mitigation via fem-based crash severity maps," in 2019 IEEE Intelligent Vehicles Symposium (IV), 2019, pp. 2187-2194.
[12] M. Parseh, F. Asplund, M. Nybacka, L. Svensson, and M. Törngren, "Pre-crash vehicle control and manoeuvre planning: A step towards minimizing collision severity for highly automated vehicles," in 2019 IEEE International Conference of Vehicular Electronics and Safety (ICVES), Sep. 2019, pp. 1-6.

[13] A. Malliaris, K. Digges, and J. DeBlois, "Relationships between crash casualties and crash attributes," SAE transactions, pp. 576-587, 1997.

[14] Association for the Advancement of Automotive Medicine, "Abbreviated Injury Scale," 2016-2019. [Online]. Available: https://www.aaam.org/ abbreviated-injury-scale-ais/

[15] M. Stevenson, M. Segui-Gomez, I. Lescohier, C. Di Scala, and G. McDonald-Smith, "An overview of the injury severity score and the new injury severity score," Injury Prevention, vol. 7, no. 1, pp. $10-13,2001$.

[16] C. A. F. Douglas W. Kononen and S. C. Wang, "Identification and validation of a logistic regression model for predicting serious injuries associated with motor vehicle crashes," Accident Analysis \& Prevention, vol. 43, no. 1, pp. 112 - 122, 2011.

[17] R. Buendia, S. Candefjord, H. Fagerlind, A. Bálint, and B. A. Sjöqvist, "On scene injury severity prediction (osisp) algorithm for car occupants," Accident Analysis \& Prevention, vol. 81, pp. 211 - 217, 2015.

[18] N. Lubbe and T. Kiuchi, "Injury estimation for advanced automatic collision notification (aacn) in germany," in 6th International Conference on ESAR; expert symposium on accident research, June 2014.

[19] T. Nishimoto, K. Mukaigawa, S. Tominaga, N. Lubbe, T. Kiuchi, T. Motomura, and H. Matsumoto, "Serious injury prediction algorithm based on large-scale data and under-triage control," Accident Analysis \& Prevention, vol. 98, pp. 266 - 276, 2017.

[20] H. Huang, C. Siddiqui, and M. Abdel-Aty, "Indexing crash worthiness and crash aggressivity by vehicle type," Accident Analysis \& Prevention, vol. 43, no. 4, pp. 1364 - 1370, 2011

[21] F. L. Mannering and C. R. Bhat, "Analytic methods in accident research: Methodological frontier and future directions," Analytic Methods in Accident Research, vol. 1, pp. 1 - 22, 2014.

[22] G. McDonald, G. Davie, and J. Langley, "Validity of police-reported information on injury severity for those hospitalized from motor vehicle traffic crashes," Traffic Injury Prevention, vol. 10, no. 2, pp. 184-190, 2009.

[23] F. Mannering, V. Shankar, and C. Bhat, "Unobserved heterogeneity and the statistical analysis of highway accident data," Analytic Methods in Accident Research, vol. 11, pp. 1-16, 2016.

[24] G. Bahouth, K. Digges, and C. Schulman, "Influence of injury risk thresholds on the performance of an algorithm to predict crashes with serious injuries," Annals of advances in automotive medicine, vol. 56, pp. 223-30, October 2012.

[25] B. Paden, M. Čáp, S. Z. Yong, D. Yershov, and E. Frazzoli, "A survey of motion planning and control techniques for self-driving urban vehicles," IEEE Transactions on intelligent vehicles, vol. 1, no. 1, pp. 33-55, 2016.

[26] J. K. Subosits and J. C. Gerdes, "From the racetrack to the road: Realtime trajectory replanning for autonomous driving," IEEE Transactions on Intelligent Vehicles, vol. 4, no. 2, pp. 309-320, June 2019.

[27] A. Liniger, A. Domahidi, and M. Morari, "Optimization-based autonomous racing of 1: 43 scale rc cars," Optimal Control Applications and Methods, vol. 36, no. 5, pp. 628-647, 2015.

[28] L. Svensson, M. Bujarbaruah, N. R. Kapania, and M. Törngren, "Adaptive trajectory planning and optimization at limits of handling," in 2019 IEEE/RSJ International Conference on Intelligent Robots and Systems (IROS), 2019, pp. 3942-3948.

[29] J. Ziegler, P. Bender, T. Dang, and C. Stiller, "Trajectory planning for bertha-a local, continuous method," in Intelligent Vehicles Symposium Proceedings, 2014 IEEE. IEEE, 2014, pp. 450-457.

[30] T. M. Howard, C. J. Green, A. Kelly, and D. Ferguson, "State space sampling of feasible motions for high-performance mobile robot navigation in complex environments," Journal of Field Robotics, vol. 25, no. 6-7, pp. 325-345, 2008.

[31] M. Werling, S. Kammel, J. Ziegler, and L. Gröll, "Optimal trajectories for time-critical street scenarios using discretized terminal manifolds," The International Journal of Robotics Research, vol. 31, no. 3, pp. 346-359, 2012.

[32] A. Rusu, S. Moreno, Y. Watanabe, M. Rognant, and M. Devy, "State lattice generation and nonholonomic path planning for a planetary exploration rover," in 65th International Astronautical Congress 2014 (IAC 2014), vol. 2, Toronto, Canada, Sep. 2014, p. 953.

[33] S. Arora, S. Choudhury, D. Althoff, and S. Scherer, "Emergency maneuver library-ensuring safe navigation in partially known environments," in 2015 IEEE international conference on robotics and automation (ICRA). IEEE, 2015, pp. 6431-6438. 
[34] Loughborough University - Vehicle Safety Research Centre (VSRC), Ergonomics and Safety Research Institute (ESRI), "Pan-European co-ordinated accident and injury databases, Pendant: Deliverable D2 - Specification of data to be collected in WP2, Specification of new accident causation data fields: Pendant Glossary \& Reference," 2012. [Online]. Available: https://trimis.ec.europa.eu/ project/pan-european-co-ordinated-accident-and-injury-database.

[35] A. Hakkert and D. Mahalel, "Estimating the number of accidents at intersections from a knowledge of the traffic flows on the approaches," Accident Analysis \& Prevention, vol. 10, no. 1, pp. 69 - 79, 1978.

[36] U.S Department of Transportation, National Highway Traffic Safety Administration, "Pre-crash scenario typology for crash avoidance research," April 2007. [Online]. Available: https://www.nhtsa.gov/sites/nhtsa.dot. gov/files/pre-crash_scenario_typology-final_pdf_version_5-2-07.pdf

[37] "Initiative for the global harmonisation of accident data," 2019. [Online]. Available: http://www.iglad.net/

[38] “Collision Deformation Classification," May 2011. [Online]. Available: https://doi.org/10.4271/J224_201105

[39] J. I. Hoffman, "Chapter 20 - odds ratio, relative risk, attributable risk, and number needed to treat," in Basic Biostatistics for Medical and Biomedical Practitioners (Second Edition), 2nd ed., J. I. Hoffman, Ed. Academic Press, 2019, pp. 295-310.

[40] J. Andersson, J. Gillis, G. Horn, J. Rawlings, and M. Diehl, "Casadi: a software framework for nonlinear optimization and optimal control," Mathematical Programming Computation, vol. 11, 072018.

[41] J. Rawlings, D. Mayne, and M. Diehl, Model Predictive Control: Theory, Computation, and Design, 2nd ed. Nob Hill Publishing, 012017.

[42] A. Wächter and L. T. Biegler, "On the implementation of an interiorpoint filter line-search algorithm for large-scale nonlinear programming," Mathematical Programming, vol. 106, no. 1, pp. 25-57, Mar 2006.

[43] M. Diehl, H. G. Bock, H. Diedam, and P.-B. Wieber, "Fast Direct Multiple Shooting Algorithms for Optimal Robot Control," in Fast Motions in Biomechanics and Robotics, Heidelberg, Germany, 2005.

[44] W. Zhang, Z. Wang, C. Zou, L. Drugge, and M. Nybacka, "Advanced vehicle state monitoring: Evaluating moving horizon estimators and unscented kalman filter," IEEE Transactions on Vehicular Technology, vol. 68 , no. 6, pp. 5430-5442, 2019.

[45] P. Polack, F. Altché, B. d'Andréa Novel, and A. de La Fortelle, "The kinematic bicycle model: A consistent model for planning feasible trajectories for autonomous vehicles?" in 2017 IEEE Intelligent Vehicles Symposium (IV), June 2017, pp. 812-818.

[46] S. Lefèvre, D. Vasquez, and C. Laugier, "A survey on motion prediction and risk assessment for intelligent vehicles," ROBOMECH Journal, vol. 1 pp. 1-14, 2014.

[47] A. Houenou, P. Bonnifait, V. Cherfaoui, and W. Yao, "Vehicle trajectory prediction based on motion model and maneuver recognition," in 2013 IEEE/RSJ International Conference on Intelligent Robots and Systems, 2013, pp. 4363-4369.

[48] A. Elfes, "Using occupancy grids for mobile robot perception and navigation," Computer, vol. 22, no. 6, pp. 46-57, June 1989.

[49] A. M. Martinez, H. Evdorides, C. L. Naing, A. Kirk, J. Tecl, J. Barrios, M. Simon, V. Phan, and T. Hermitte, "Accident causation and preaccidental driving situations. Part 3. Summary report,” Loughborough University, Loughborough, U.K., Tech. Rep., 2008.

[50] J. Mårtensson, M. Nybacka, J. Jerrelind, and L. Drugge, "Evaluation of safety distance in vehicle platoons by combined braking and steering," in Proceedings of 11th International Symposium on Advanced Vehicle Control, Sept 9-12, 2012, Seoul, Korea. Japan Society of Mechanical Engineers (JSAE), 2012.

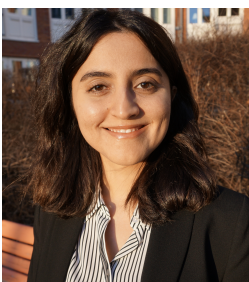

Masoumeh Parseh received her M.Sc. degree in mechanical engineering from Babol University of Technology, Babol, Iran, in 2015. She is currently pursuing a Ph.D. degree in the Mechatronics Division at KTH Royal Institute of Technology in Stockholm, Sweden. Her research is focused on safety analysis for autonomous vehicles in critical situations.

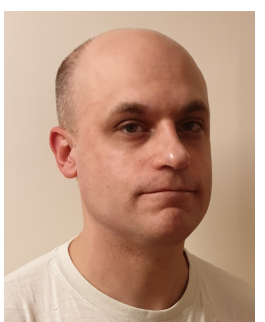

Dr. Fredrik Asplund is an assistant professor of safety practice at KTH Royal Institute of Technology. Dr. Asplund is a Marie Curie Fellow and a VINN MER Fellow. His research interests include system safety, safety standards, safety-security interactions, empirical software engineering, innovation, and engineering education in the Cyber-Physical Systems domains.

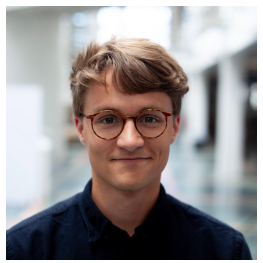

Lars Svensson received his B.Sc and M.Sc degrees in Engineering Physics from Uppsala University, Uppsala, Sweden in 2015 . He is currently pursuing a Ph.D at the Mechatronics Division at KTH Royal Institute of Technology in Stockholm, Sweden. During the fall of 2018, he was a visiting researcher at the Model Predictive Control Lab at University of California, Berkeley. His research focuses on motion planning of road vehicles in critical situations.

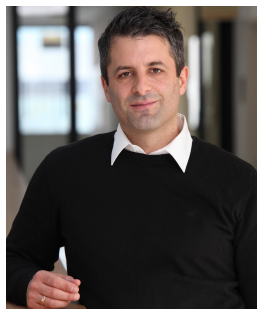

Wolfgang Sinz [Ph.D., (M)] is a Senior Scientist at the Vehicle Safety Institute at TU Graz. He is habilitated in the subject of Transport Safety. In addition to the analysis of the crash behavior of high-voltage batteries, his main research field is the assessment of integrated vehicle safety systems for crash avoidance and injury mitigation.

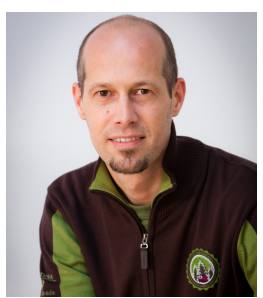

Ernst Tomasch [Ph.D., (M)] is a Senior Scientist at the Vehicle Safety Institute at TU Graz. His main activities include the analysis and reconstruction of road accidents and database analysis. He is also working on the effectiveness assessment of advanced driver assistance systems whereby the in-house indepth accident database CEDATU serves as the basis for the virtual assessment.

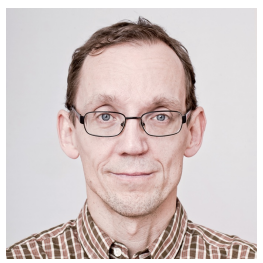

Martin Törngren has been a professor of embedded control systems at KTH Royal Institute of Technology since 2002. Networking and multidisciplinary research have been characteristic throughout his career, encompassing control and embedded systems codesign, and engineering methodologies for autonomous trustworthy cyber-physical systems. He has spent several periods abroad as a visiting scholar including 2011/12 at UC Berkeley, spring 2018 at Stevens Institute of Technology, and fall 2018 at UC Berkeley. He is the principal initiator and director of the Innovative Centre for Embedded Systems (ICES), a KTH-industry competence network launched in 2008 . 